\title{
Results on intense beam focusing and neutralization from the neutralized beam experiment
}

\author{
P. K. Roy, S. S. Yu, S. Eylon, E. Henestroza, A. Anders, F. M. Bieniosek, W. G. Greenway, B. G. Logan, W. L. \\ Waldron, and D. L. Vanecek \\ Lawrence Berkeley National Laboratory, 1 Cyclotron Road, Berkeley, CA-94720, USA \\ D. R. Welch and D. V. Rose \\ Mission Research Corporation, 5001 Indian School Rd NE, Albuquerque, NM 87110-3946, USA \\ R. C. Davidson, P. C. Efthimion, E. P. Gilson, and A. B. Sefkow \\ Princeton Plasma Physics Laboratory, Princeton, NJ 08543-0451, USA
}

\author{
W. M. Sharp \\ Lawrence Livermore National Laboratory, 7000 East Ave., L-645, Livermore, CA 94550, USA
}

We have demonstrated experimental techniques to provide active neutralization for space-charge dominated beams as well as to prevent uncontrolled ion beam neutralization by stray electrons. Neutralization is provided by a localized plasma injected from a cathode arc source. Unwanted secondary electrons produced at the wall by halo particle impact are suppressed using a radial mesh liner that is positively biased inside a beam drift tube. We present measurements of current transmission, beam spot size as a function of axial position, beam energy and plasma source conditions. Detailed comparisons with theory are also presented.

\section{INTRODUCTION}

Final focusing has been a subject of intense study [13] from the very early days of heavy ion fusion (HIF). Neutralized ballistic transport (NBT) [4-11] is presently being studied for propagating intense heavy ion beams inside a reactor chamber to an inertial confinement fusion (ICF) target. A recent HIF driver study [12] demonstrates that stringent final-focus requirements [13-15] can be met provided that active neutralization is implemented to overcome the formidable space charge of the intense ion beams. Other beam transport schemes under consideration include self-pinched transport [16-20] and discharge channel [21-23] transport.

In the NBT scheme, the individual beams focus outside of the target chamber and enter through ports in the chamber walls. These beams are focused and directed such that they intersect before striking the target and then strike the target as they are expanding into an annular configuration [24]. The target chamber is filled at low pressure with a gas such as flibe. A volumetric plasma is produced as the flibe gas is partially ionized by the beam as well as by xrays emitted by the hot target.

The volumetric plasma is not adequate to provide the necessary neutralization. Therefore, additional plasma, the "plasma plug," is externally injected near the chamber entry port, through which the beam passes. Chamber transport using annular and solid plasma regions in the transport chamber has been examined numerically by several investigators $[17-18,25]$. The general concept studied in this paper consists of an initially-non neutralized beam passing through a finite thickness of plasma and dragging along plasma electrons for partial charge and current neutralization.

An earlier experiment [26] examined the charge neutralization of a heavy ion beam by electrons drawn from a localized source as the beam was focused. The electron source was a glowing tungsten filament placed in the beam path, enabling the supply of thermionicallyemitted electrons inside of the beam. The experiment demonstrated the beneficial effect of charge neutralization on a heavy-ion beam, and these results were confirmed in a series of electrostatic particle-in-cell (PIC) simulations.

To quantitatively ascertain the various mechanisms for neutralization, the Neutralized Transport Experiment (NTX) was constructed at Lawrence Berkeley National Laboratory. In this experiment a high quality beam is passed through well-characterized plasma sources. The objective is to provide sufficently detailed experiment data to validate simulation code predictions. Here, we are presenting initial results of neutralization from localized plasma plug on the NTX. This article describes the neutralization physics, NTX beamline system, techniques to control stray electrons in vacuum transport, and beam neutralization using a plasma plug.

\section{PHYSICS OF NEUTRALIZATION}

The plasma plug provides electrons that neutralize to $>90 \%$ the charge of a convergent beam. Typically, $n_{p} / Z n_{b}$ $>1$, where $n_{p}$ is the plasma density and $n_{b}$ and $Z$ are the ion beam density and charge state. Ideally, the plasma is in electrical contact with a conducting boundary at large radius enabling a continuous supply of electrons. Stationary plasma can only provide an ion beam electron neutralization down to some minimum space-charge potential. The key scaling parameter for beam transport is the dimensionless perveance defined as the ratio of the beam space charge to kinetic energy $\left(K=2 I_{b} / I_{A} \beta_{i}{ }^{2}\right.$, where $I_{A}=\beta_{i} \gamma_{i} m_{i} c^{3} / e Z$ is the Alfven current with a beam of current $I_{b}$, velocity $\beta_{i} c$, and relativistic factor $\gamma_{\mathrm{i}}$ ). Provided $K m_{i} / Z m_{e}>1$, electrons from this plasma can accelerate in 
the beam space-charge potential to the beam velocity. This condition limits the minimum residual space charge potential to $1 / 2 \mathrm{~m}_{\mathrm{e}} \mathrm{v}_{\mathrm{i}}^{2}$ [27]. Previous neutralization experiments $[26,28]$ have provided, to some degree, confirmation of this limit.

Plasma neutralization in NTX was simulated with the PIC code LSP [13-14]. The low emittance ( $\varepsilon \sim 30 \pi \mathrm{mm}-\mathrm{mr})$ of the NTX beam at the entrance to the neutralized region allows for the beam to be focused to a small spot (1-2mm radius). Several $r-z$ LSP simulations were run using the NTX geometry with a nominal 255-keV, 24-mA singly charged potasium ion beam assuming the beam envelop is circular. The beam enters the neutralization pipe $(\mathrm{z}=0)$ with a 2 -cm outer radius and a 20-milliradian convergence angle. Figure 1 shows the beam envelope radius for 3 simulations with: perfect neutralization (ballistic), no neutralization (vacuum), and a MEVVA source generated plasma (plasma plug) descibed in next section with a maximum $10^{10} \mathrm{~cm}^{-3}$ density. With no neutralization, the simulation gives a $1.64 \mathrm{~cm}$ radius at this distance. With the perfect neutralization, we calculate yields a $1-\mathrm{mm}$ RMS spot at focus $(z=100 \mathrm{~cm})$. Including the MEVVA plasma yields a spot only slightly larger than ballistic $(1.35 \mathrm{~mm}$ at $\mathrm{z}=100 \mathrm{~cm})$. In this case, the plasma electrons provide a source of co-moving electrons with a $96 \%$ effective neutralization.

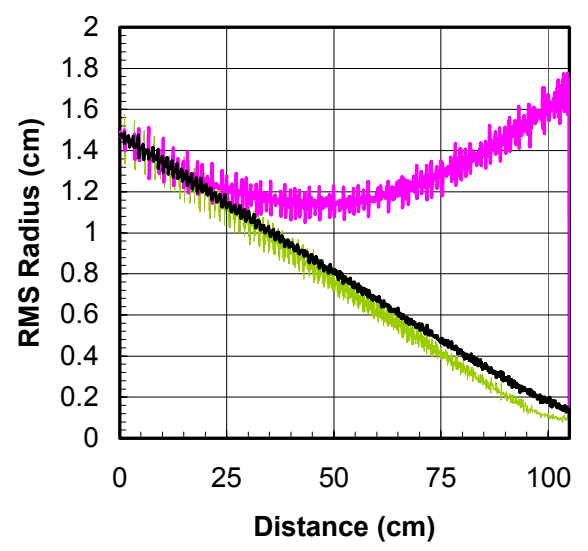

FIG. 1. A comparison of beam envelop for simulations with: perfect neutralization (the lower line), no neutralization or vacuum (the top line) and MEVVA source or plasma plug (middle line).

\section{DESCRIPTION OF NTX BEAMLINE}

NTX consists of three major sections: a potassium source chamber [29], a magnetic transport section with four pulsed quadrupoles [30], and a one-meter long neutralization drift section with plasma plug [31]. Figure 2 shows a sketch of the NTX beamline. A thorough description of the design and characterization of this NTX beam line has been submitted recently for publication[32]. We now describe the major sections of NTX.

\section{A. Ion source}

The $\mathrm{K}^{+}$beam is produced on a standard hot-plate source [33], with the perveance being determined by passing the beam through a metal aperture after the diode. Pulsed power is provided by a Marx generator that was used in the Multiple Beam Test Experiment (MBE-4) [34]. A timed crowbar switch on NTX produces pulses with 0.5 --- $1 \mu$ s rise time and a $10-\mu$ s "flat-top".

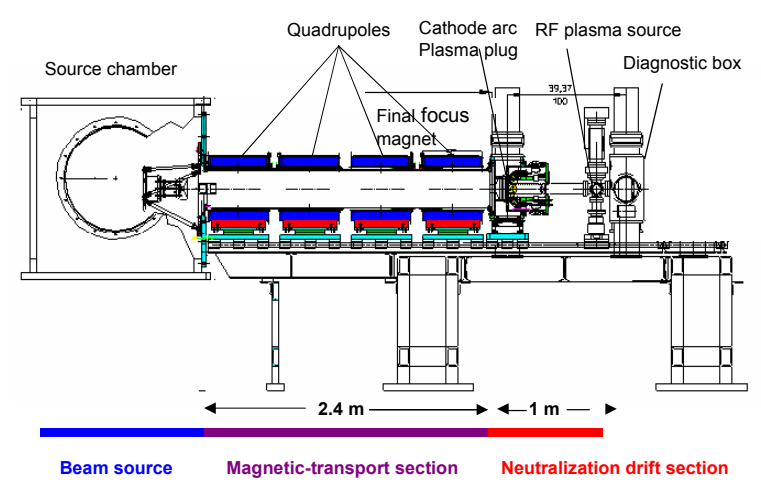

FIG. 2. A schematic of NTX beamline setup.

\section{B. Magnetic Beam transport}

The section consists of four pulsed quadrupole magnets separated by short drift regions. The quadrupole fields are chosen to obtain a beam with 1-m focal length (20-mm radius and $20-\mathrm{mr}$ convergence angle) at the entrance to the neutralization region. The choice of a 60$\mathrm{cm}$ half-lattice period and 2.4-m total length is a scaled version of a driver design.

\section{Plasma source and focusing section}

Figure 3 shows (a) schematic of a 1-m long neutralization section indicated the location of the different plasma sources (b) the neutralization section on NTX and (c) the cathode arc plasma source. We now present results using cathode arc plasma source referred to as the MEVVA plasma plug throughout the article. The plasma density of the MEVVA plasma plug can be estimated by noting that the ion current is given generally by $j_{i}=z e n_{i} v_{i}$, where $j_{i}$ is the ion current density, $z$ is the average charge state number (1.7), $e$ is the elementary charge, $n_{i}$ is the ion number density, and $v_{i}$ is the average ion velocity $\left(1.54 \times 10^{5} \mathrm{~m} / \mathrm{s}\right)$ in the direction of the collector, which is here identical with the plasma flow velocity. With an area of collection of about $10^{-2} \mathrm{~m}^{2}$, one obtains $n_{i} \approx 1.8 \times 10^{10} \mathrm{~cm}^{-3}$ for the average plasma density inside the metal shield at about $250 \mu$ s after arc triggering, at a pulse-forming network (PFN) charging voltage of $2.0 \mathrm{keV}$. We find that the NTX cathode-arc source produces plasmas with densities in the $10^{10}-10^{11}$ $\mathrm{cm}^{-3}$ ranges and that the plasma density is proportional to the discharge voltage up to $2.5 \mathrm{keV}$.

\section{Optical imaging technique for beam profile}

Non neutralized and neutralized beam were recorded using modern optics. We have used glass and ceramic (98\% alumina) as scintillator materials. Charge neutralization was provided by a high-transparency (80- 
$90 \%$ transmission) metallic mesh placed on or near the surface of the scintillator. By applying a negative bias to the mesh, stray external electrons were decelerated and deflected away from the scintillator, limiting their contribution to the optical image to negligible levels. Time-resolved beam-induced images on the scintillator screen were captured with a Roper Scientific gated intensified CCD camera viewing the scintillator through a vacuum window, and images are processed using the public-domain program ImageJ.

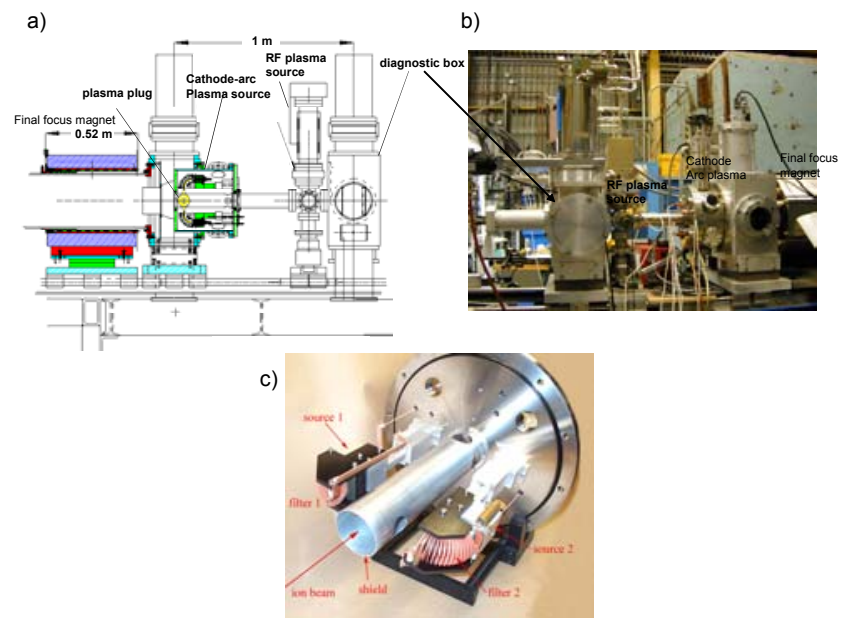

FIG. 3. (a) Schematic of $1 \mathrm{~m}$ long neutralization section with location of the plasma sources (b) viewgraph of the schematic and (c) viewgraph of cathode are plasma source.

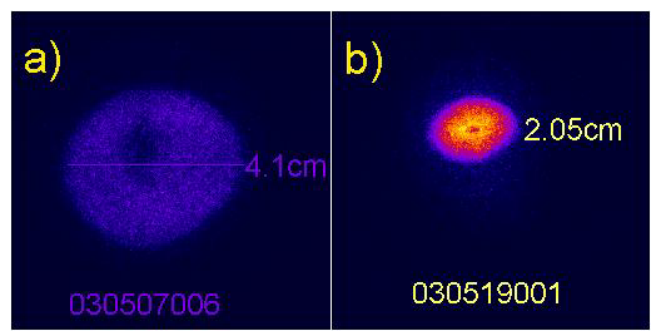

Fig.4. Beam images for a $255 \mathrm{keV}$ beam measured $1 \mathrm{~m}$ downstream, transported through a tube of diameter (a) $15 \mathrm{~cm}$ and b) $7.6 \mathrm{~cm}$.

\section{BEAM TRANSPORTATION IN VACUUM \\ A. Uncontrolled neutralization}

As a preliminary step to characterize beam transport in the NTX final-focus system, a $255-\mathrm{keV}$ beam was injected into a $15-\mathrm{cm}$ diameter pipe from the exit of the final focus magnet to $1 \mathrm{~m}$ downstream, ensuring that electron emission from the walls was negligible. Later, in order to use the beam with a matching system of the MEVVA plasma plug and the final focus drifting section, it was transported through a nominal $7.6-\mathrm{cm}$ diameter beam. Figure 4 shows beam images for a beam transported through the (a) $15 \mathrm{~cm}$ and (b) $7.6 \mathrm{~cm}$ diameter tube. A smaller spot size, roughly $50 \%$ less in diameter, was measured for transport in the $7.6 \mathrm{~cm}$ diameter tube, which did not agree well with the calculated beam transport in a vacuum. This smaller size was due to the capture by the beam of free electrons from the wall that partially neutralized the beam.

\section{B. Control technique of uncontrolled neutralization}

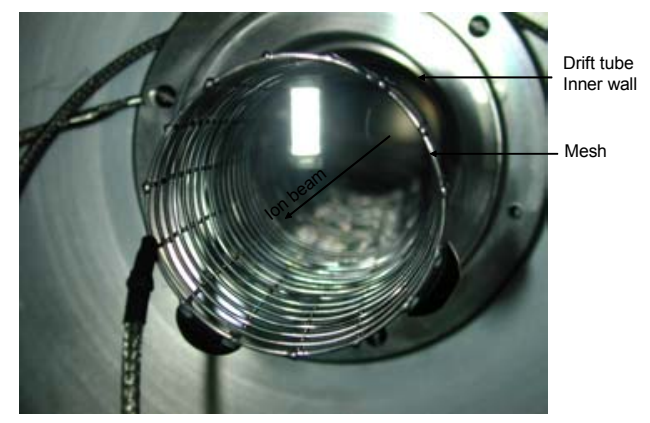

FIG.5. Cylindrical metal mesh of outer diameter $6.3 \mathrm{~cm}$ was installed inside a $7.6 \mathrm{~cm}$ diameter beam drift tube.

Ions from the poorly matched beam front and halo ions in the main pulse of the beam can strike the outer wall of the transport tube. A single ion impact can produce thousands of secondary electrons depending on the energy and angle of incidence [35-36] with ions of grazing-angle incidence producing the largest secondary electron yield [37]. Only a small fraction of the beam ions striking the wall are needed to provide a space-charge limited supply of electrons from the wall. If the secondary electrons are not stopped, they are attracted to the beam potential and can provide some degree of beam neutralization. Halo scrape off will be drastically reduced using the $15 \mathrm{~cm}$ pipe. Also for larger wall radius, the wall electrons can spend only a small fraction of their time within the beam. The electrons are moving at their greatest velocity while passing through the beam further decreasing their beam neutralization. Thus, the neutralization fraction from these electrons will scale no better than the ratio of the beam to wall radii. A wall radius comparable to that of the beam will provide some sizable degree of neutralization and prevent the observation of expected "vacuum transport." The secondary electrons are produced with mean energy roughly that of the ionization potential of the impacted wall atoms - typically $10 \mathrm{eV}$. The distribution of electrons in ionization events also has a high energy tail falling off as the square of the energy. Thus, if we place a highly transparent wire mesh sleeve within the drift tube and bias it with potential $>10 \mathrm{eV}$, we can expect to collect these secondary electrons and prevent them from moving into the beam path. Given a positive potential, electrons produced on the mesh itself will tend to be trapped near the mesh. Figure 5 shows a high beam transference cylindrical tube shape metal mesh that was 
inserted into the $7.6 \mathrm{~cm}$ beam tube. The thickness and longitudinal length of the mesh were $2.2 \mathrm{~mm}$, and 58.2 $\mathrm{cm}$, respectively. Outer and inner diameters of the mesh tube were $6.3 \mathrm{~cm}$ and $5.88 \mathrm{~cm}$, respectively, thus maintaining better than a $5 \mathrm{~mm}$ radial electrical isolation from the beam tube wall. Figure 6 shows a pattern of beam profiles corresponding to energies for vacuum transport in (a) WARP calculation, the (b) $15 \mathrm{~cm}$ diameter tube and (c) $7.6 \mathrm{~cm}$ diameter tube using the mesh bias of positive $1 \mathrm{keV}$. Using the mesh bias, the measured beam profile was in general agreement with WARP for vacuum transport. Figure 7 shows the measured beam profile for varying mesh bias. In Fig. 7(a), the lower line with solid circles shows that a beam diameter of $2.4 \mathrm{~cm}$ was measured with $0 \mathrm{~V}$ across the mesh bias for $255-\mathrm{keV}$ beam energy. A beam diameter of roughly $3.75 \mathrm{~cm}$ was also measured by applying $\pm 500 \mathrm{~V}$ across the mesh for the same beam energy, shown by lines of solid diamonds and cross symbols, respectively. The line with hollow circles shows a measured beam diameter of $3.75 \mathrm{~cm}$ using a mesh bias of $+250 \mathrm{~V}$. A larger beam diameter of $4 \mathrm{~cm}$ was measured with a mesh bias of $\pm 1 \mathrm{keV}$ for the same 255 $\mathrm{keV}$ beam energy, as shown by lines of hollow diamond and solid triangle symbols in the figure. The positive 250 $\mathrm{V}$ bias on the mesh provides a smooth trend of beam shape, regardless of beam energies in the range of 245 to $300 \mathrm{keV}$.

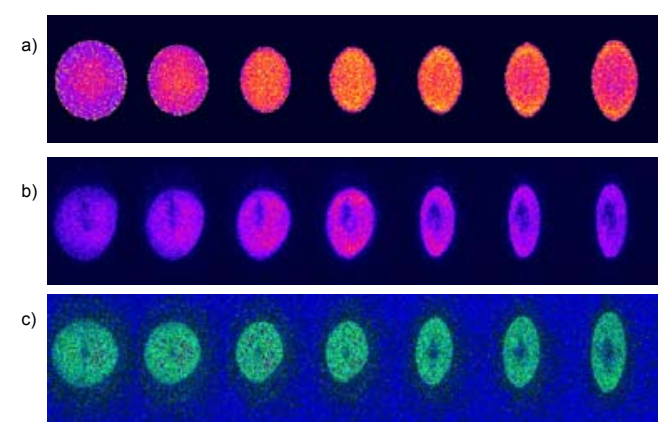

Fig.6. Beam profile for vacuum transport for $240-310 \mathrm{keV}$ beam energies from (a) WARP calculations (b) experimental measurements for transport through a $15 \mathrm{~cm}$ diameter tube, and (c) experimental measurements for transport through a $7.6 \mathrm{~cm}$ diameter tube using mesh bias of $+1 \mathrm{keV}$.

Beam diameter measurement by varying beam energies was performed in a $15 \mathrm{~cm}$ diameter vacuum tube separately, where the possibility of a wall-electron effect was negligible. There was no mesh or plasma inside the tube that could influence measurements of ion beam transport in vacuum conditions. Figure 7(c) shows a comparison of beam diameters for transportation through the mesh embedded $7.6 \mathrm{~cm}$ diameter tube with a bias of $+250 \mathrm{~V}$ and $15-\mathrm{cm}$ diameter vacuum tube. The dotted lines with hollow circles and triangles represent beam diameters that were measured in the $\mathrm{x}$ and $\mathrm{y}$ axis, respectively, for a beam of energies 240 to $310 \mathrm{keV}$ transported through the $15-\mathrm{cm}$ diameter tube. Diameters of $4.53,4.0$, and $2.68 \mathrm{~cm}$ were measured in the $\mathrm{x}$-axis for the beam of energies 259, 268 and $298 \mathrm{keV}$, respectively. On the other hand, the lines with solid circles and triangles represent beam diameters that were measured in the $\mathrm{x}$ and $\mathrm{y}$ axis, respectively, for a beam of energies 244 to $290 \mathrm{keV}$ transported through the $7.6 \mathrm{~cm}$ diameter tube. Beam diameters of $3.76,3.15$, and $2.41 \mathrm{~cm}$ were measured in the x-axis for the beam of 255, 268 and $287 \mathrm{keV}$, respectively. These were end-to-end measurements of a beam image, without the deduction of any cut off value that was used for statistical error reduction in Section-V. For a $255-\mathrm{keV}$ beam, a difference of $6 \mathrm{~mm}$ in beam diameter was measured between the two cases. This difference was smaller for a more energetic beam. For example, for a $288 \mathrm{-keV}$ beam, a difference of 2 $\mathrm{mm}$ in diameter was measured for the two cases. For a higher energy beam (say $300 \mathrm{keV}$ ), the radial distance of the beam from the wall was larger than the lower energy beam $(255 \mathrm{keV})$ and neutralization was not significant. By using the mesh and an appropriate voltage across it, we were still achieving a slightly smaller size than "expected" for an un-neutralized beam. The difference in the two cases, as we inferred, was due to the $58.2 \mathrm{~cm}$ mesh liner in the $7.6 \mathrm{~cm}$ diameter tube was not long enough to cover the entire 1-m long drift tube. As a result, partial neutralization occurred beyond the ends of the mesh. However, the mesh was a significant development in overcoming uncontrolled neutralization of wall electrons.

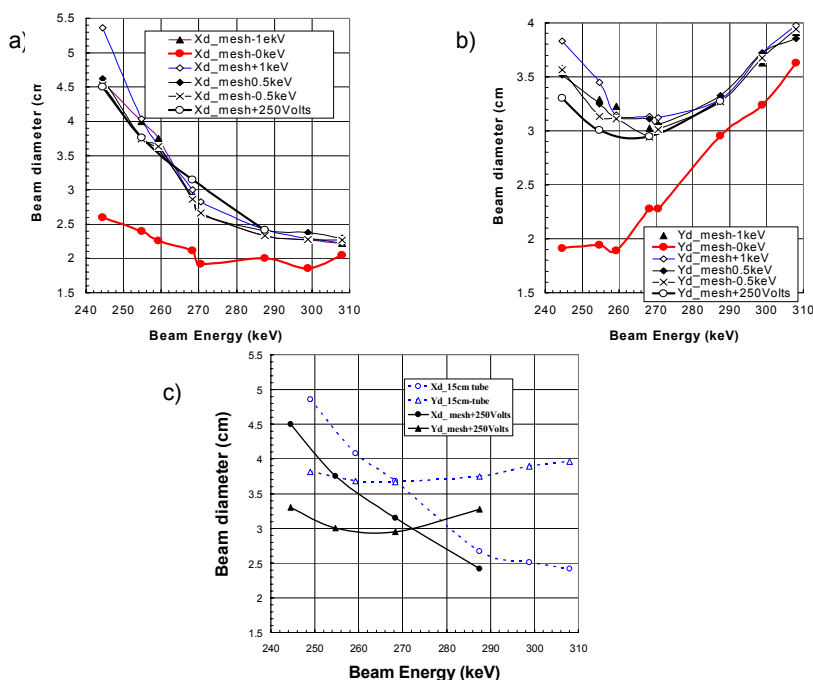

FIG.7. Beam diameters corresponding to beam energies were measured in the (a) $\mathrm{x}$-axis and (b) $\mathrm{y}$-axis by varying mesh bias, and (c) a comparison of beam size for a 255$\mathrm{keV}$ beam transported through a $15-\mathrm{cm}$ tube (dotted lines for the $x-y$ axis) and mesh included $7.6 \mathrm{~cm}$ diameter tube (solid lines for the $\mathrm{x}-\mathrm{y}$ axis) with bias $250 \mathrm{~V}$.

Currents corresponding to positive and negative voltages across the mesh were measured during $255 \mathrm{keV}$ beam pulse. Figure 8 shows experimental data of currents measurements in the mesh. A negative current of $6.56 \mathrm{~mA}$ 
was measured at a zero potential across the mesh, that shows that secondary or wall electrons movement was exist and only that electrons which were directly striking the mesh wire, were measured and inferred that uncollected electrons remaining around the mesh. A positive $50 \mathrm{~V}$ potential was applied across the mesh to collect these all electrons, a current of negative $30.72 \mathrm{~mA}$ was measured. Voltages such as negative $250 \mathrm{~V}$ and negative $500 \mathrm{~V}$ were also applied to the mesh; however, no significant current was measured. It was inferred that a higher negative potential, like negative $250 \mathrm{~V}$, across the mesh was able to completely stop radial inward and outward motion of electrons, but leaving uncollected electrons. Therefore, collection of all the electrons around the mesh, using a $+250 \mathrm{~V}$ potential, was a better choice. However, the presence of a higher mesh bias has some effect of the physics of plasma neutralization. For a positive potential, plasma electrons initially accelerated up to the beam velocity as they leave the plasma, are then accelerated up to an energy corresponding the mesh bias. The quiescent co-moving plasma electrons now have a velocity many times that of the beam. As the mesh potential increases, these electrons become more inefficient at neutralizing the beam potential and a larger beam focal spot is expected. For a negative potential, the plasma plug electrons are largely excluded from the beam in the region of the mesh yielding no neutralization.

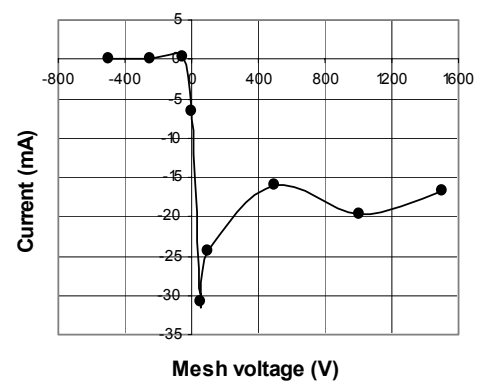

FIG. 8. Current in mesh varying mesh bias during a 255$\mathrm{keV}$ beam pulse.

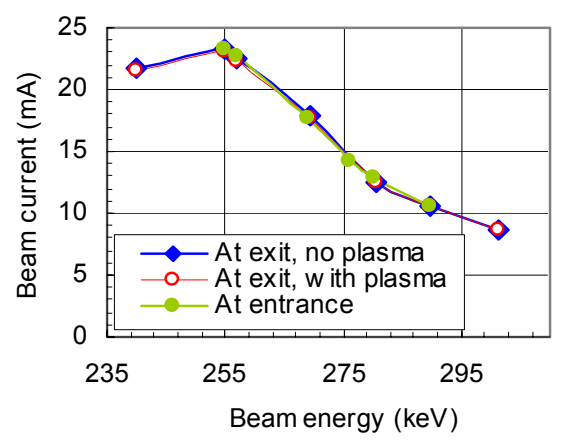

Fig.9. Measured beam current. The solid circles are the symbols of currents, measured at the entry of the $1 \mathrm{~m}$ final focus section or on the other way at the exit of the magnets. The solid diamonds in the line are the symbols of currents, measured at the end of the $1 \mathrm{~m}$ drift section and the symbol hollow circles represent MEVVA plasma plug neutralized beam current.

\section{BEAM NEUTRALIZATION \\ A. Beam current}

Beam current was measured at the exit of final focus (at diagnostic box in the Fig. 3a) with and with out plasma, and at the entrance of final focus (at the end of final focus magnet). In order to measure beam current, a Faraday cup was used. The cup was biased with a $+500 \mathrm{~V}$ and its internal guard ring was biased with a negative 900 V. An electron trap was installed in front of the Faraday cup. Figure 9 shows beam currents as a function of energies for beam aperture at the entrance of neutralization drift section. All measurements overlap each other and show 100\% beam current transport in the system. There was no significant beam loss in the drift section, and full beam current was transported before neutralization and during neutralization.

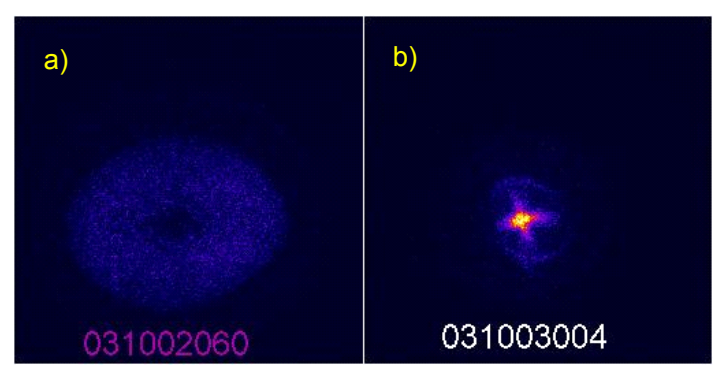

FIG. 10. Beam images for a non-neutralized (left) and neutralized (right) beam of energy $255 \mathrm{keV}$. In both cases beam was transported through a $7.6-\mathrm{cm}$ diameter tube (mesh bias was $+250 \mathrm{~V})$.

\section{B. Neutralized beam}

The ion beam was neutralized using plasma electrons from the MEVVA plasma (describe in the section IIIC). A series of neutralized beam spot size measurements is under way with various conditions and parameters. Figure 10 shows viewgraph of beam images for (a) non neutralized and (b) neutralized beam of energy $255 \mathrm{keV}$. In both cases the beam was transported through a 7.6-cm diameter tube (mesh bias was $+250 \mathrm{~V}$ ). The RMS beam radius (using higher and lower cut-off values) of the non neutralized beam was $14.7 \mathrm{~mm}$ (end-to-end eye view RMS radius was $16.4 \mathrm{~mm}$ ), and the RMS radius of the neutralized beam was $1.26 \mathrm{~mm}$. These measurements shows that a vacuum transport beam was compressed approximately $90 \%$ of its volume by beam neutralization.

Variation of neutralized beam radius with axial position was measured at the diagnostic box by varying scintillator position over a range of around $15 \mathrm{~cm}$. Figure 11 shows axial beam envelope variations in (a) an experiment using mesh with $+250 \mathrm{~V}$ inside the $7.6 \mathrm{~cm}$ drift tube and (b) theoretical calculation without mesh consideration. Though the discrepancy in beam radius is less than a millimeter, this difference might be due to the absence of mesh in the calculation. However, we are very 
close to understand neutralized beam envelope for final focus.
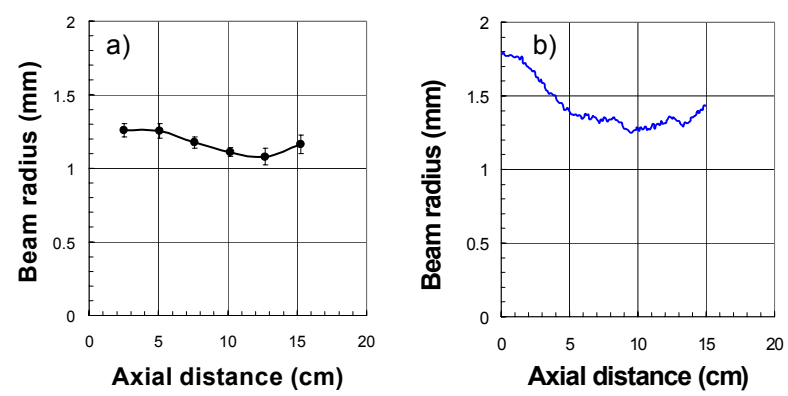

FIG.11. Axial beam envelope variations in the (a) $7.6 \mathrm{~cm}$ drift tube (mesh bias with $+250 \mathrm{~V}$ ) and (b) theoretical calculation (without mesh). a)

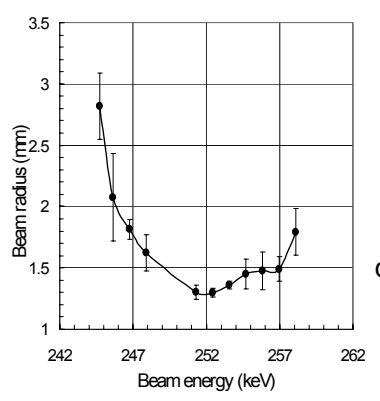

b)

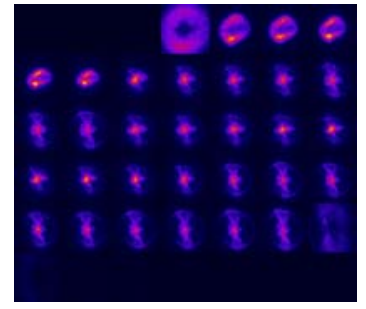

c)

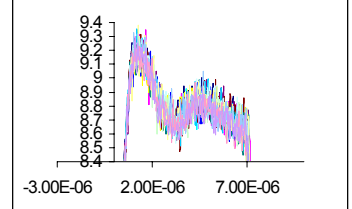

FIG.12. shows (a) variation of neutralized beam radius corresponds to beam energies (b) beam pattern from head to tail by varying time delay of image recording, (c) variation of multiple Marx waveforms for a same condition.

The radius of the neutralized NTX beam was also measured for various beam energies, which was produces using the Marx crowbar pulse system, and for various time delays in image recording. Figure 12 shows (a) variation of neutralized beam radius corresponds to beam energies (b) beam pattern from head-to-tail by varying time delay of image recording, (c) variation of multiple Marx waveforms for a same condition. For the energy variation, sensitivity to chromatic variation is a result of magnetic quadrupole optics. For the head to tail observation setup was that a beam of $255 \mathrm{keV}$ was transported through a mesh-less $7.6 \mathrm{~cm}$ diameter tube and plasma was with $2 \mathrm{keV}$ discharge potential. The time slice of width was $100 \mathrm{nsec}$ with gain setup at 253 and delays between 4.6 and $12.8 \mu \mathrm{sec}$ was at intervals of $0.2 \mu \mathrm{sec}$. It was observed that the beam head and tail has halos, and we inferred that the longitudinal forces of beam charges inside the beam were increasing the current at the head of the beam and slowing the tail of the beam. Though the main beam stream looked flat for the time delay of 6 to $11 \mu \mathrm{sec}$, however, an extended scaling of main beam size was showed that main beam stream was varied sometimes a fraction of $\mathrm{mm}$. This might be due to shot to shot variation of Marx voltage as shown waveforms in the Fig. 12(c), or variation of charges accumulation in the capacitor tank of the MEVVA plasma plug high voltage system.
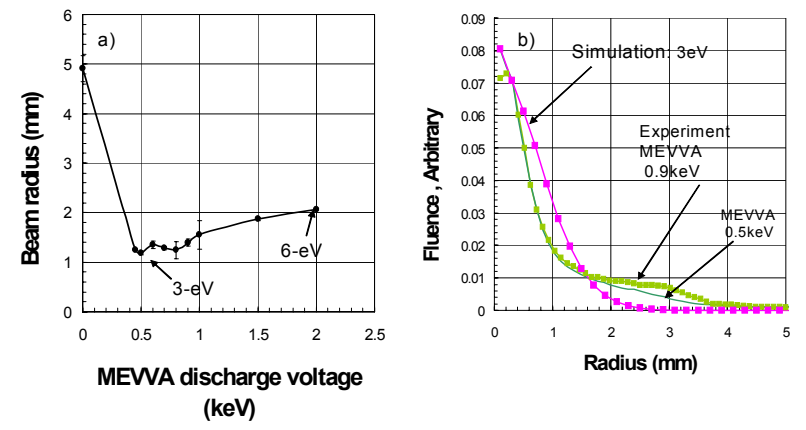

FIG. 13. (a) Neutralized beam radius vs plasma discharge voltage, (b) comparison between experiment and theory of the radial distribution profile.

The neutralized beam radius was also varied with MEVVA plasma discharge voltages. Figure 13 shows (a) beam radius vs plasma discharge voltage, and (b) comparison between experiment and theory of the radial distribution profile. The basic size of the beam spot is similar in both cases (experiments and theory) with differences attributed to a halo due to nonlinear focusing seen in the experimental curve. Simulations show that if electrical connection is maintained to the pipe wall through electron space charge limited emission (SCLE), the beam spot shows little variation for plasma densities ranging from $3 \times 10^{8}-3 \times 10^{10} \mathrm{~cm}^{-3}$ for an initial plasma temperature of $3-\mathrm{eV}$. For a $6-\mathrm{eV}$ initial plasma temperature, which is greater than $1 / 2 \mathrm{~m}_{\mathrm{e}} \mathrm{v}_{\mathrm{i}}^{2}$, the beam spot size was roughly $50 \%$ larger than the case with $3-\mathrm{eV}$ plasma (Fig. 13a). The sensitivity of the beam spot to incoming beam emittance is calculated to be weak with only a $30 \%$ spot-size variation for a factor of three change in emittance. This low sensitivity to emittance indicates that charge neutralization in the NTX experiment should be quite close to that $96 \%$ value seen in simulations and not influenced by details in the emittance.

\section{Neutralized and Ballistic Beams}

The Neutralized NTX beam was compared with an array of pinhole beamlets. Since the pinhole beam has a negligible space charge and emittance, its trajectory is effectively ballistic. Each beam let was formed by passing the beam through a cross slits system. We used the MEVVA plasma source to neutralize the full-current NTX 
beam. Figure 14 shows comparison of (a) line integral profile of experimental ballistic beam and plasma neutralized beam, (b) LSP theoretical simulation for the beams (c) experimental ballistic beam image, and (d) MEVVA neutralized beam image. There is a good agreement in theory and experiments. Though we observed the profile of neutralized beam is slightly larger than the pinhole beam image, this was an effect of the residual space charge. a)

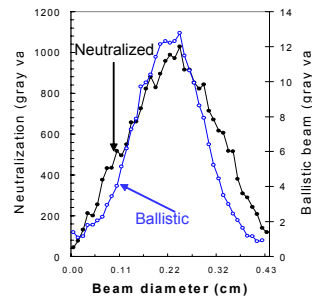

b)

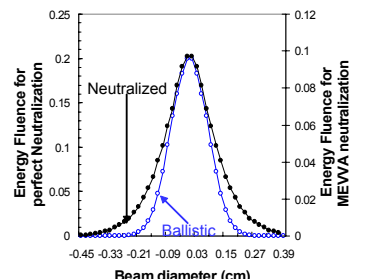

c)

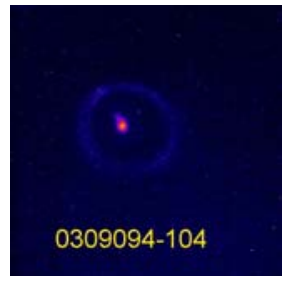

d)

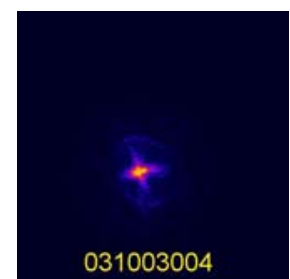

FIG. 14. A comparison of (a) line integral profile of experimental ballistic beam and plasma neutralized beam, (b) LSP theoretical simulation for the beams (c) experimental ballistic beam image, and (d) MEVVA neutralized beam image.

\section{CONCLUSION}

Several experiments have recently been carried out on the Neutralized Transport Experiment (NTX) at Lawrence Berkeley National Laboratory. We have demonstrated experimentally that a biased cylindrical mesh inside a drift tube can prevent uncontrolled neutralization of a space-charge-dominated ion beam. Without neutralization, the NTX beam radius at the nominal 1-m focal distance is found to be $14.7 \mathrm{~mm}$ with the mesh, compared with about $10 \mathrm{~mm}$ without, in better agreement with the 16.4-mm value found in numerical simulations. When the NTX beam is neutralized by passing it through a MEVVA plasma, the focal radius decreases to 1.26 to $1.4 \mathrm{~mm}$, compared with the $1 \mathrm{~mm}$ spot size found in simulations for perfect neutralization. Another recent experiment has shown that the variation of the nonneutralized NTX beam radius with axial position near the focal point qualitatively matches theoretical predictions. Finally, good agreement is found between the radial fluence profile of a neutralized NTX beam and an effectively ballistic beam made by passing the full NTX beam through a pinhole.

\section{ACKNOWLEDGMENTS}

The authors are grateful to all scientific members and technical staff of the Heavy Ion Fusion Virtual National Laboratory (HIF-VNL). This work was performed under the auspices of the US Department of Energy by University of California Lawrence Livermore National Laboratory and Lawrence Berkeley National Laboratory under Contracts No. W-7405-ENG-48 and DE-AC-3$76 \mathrm{SF} 00098$.

\section{REFERENCES}

[1] W. B. Herrmannsfeldt, LBL-5543 (1976).

[2] S. S. Yu et al., Proc. 1978 Heavy Ion Fusion Workshop, ANL report 79-41 (1979), p. 403.

[3] C. L. Olson, LBL-10301 (1980).

[4] Barboza, N., Fusion Eng. Design 32-33, 453(1996).

[5] Callahan, D. A., Fusion Eng. Design 32-33, 441(1996).

[6] Logan, B. G. et al., Nucl. Instrum. Meth. Phys. Res A 415, 468 (1998).

[7] Vay, J.-L. et al., eds., Charge compensated ion beam propagation in a reactor sized chamber. Laser Interaction and Related Plasma Phenomena, AIP Conf. Proc.406,p. 267,NY(1997).

[8] Sharp, W. M. et al., Bulletin of the American Physical Society 44, 201(1999).

[9] Kaganovich, I. D. et al., Phy. of Plasmas 8, 4180 (2001).

[10] Rose, D. V. et al., Nucl. Instrum. Meth. Phys. Res. A 464, 299-304 (2001).

[11] Welch, D. R. et al. (2001). Nucl. Instrum. Meth. Phys. Res. A 464, 134-139.

[12] S. S. Yu et al., Proc. 2002 Amer. Nucl. Soc. Fusion Topical Meeting, 2002.

[13] T. P. Hughes, et al., Phys. Rev. ST Accel. Beams 2 (1999) 110401.

[14] D. R. Welch, et al., Nucl. Instrum. Meth. Phys. Res. A 464, 134 (2001).

[15] W. M. Sharp, et al., Proc. Particle Accel. Conf., 2003.

[16] Hahn, K. et al., Fusion Engineering Design 32-33, 417(1996).

[17] Olson, C. L. et al., Proc. Sixteenth Int. Atomic Energy Agency Fusion Energy Conf., 195. (1996).

[18] Welch, D. R. et al., Fusion Eng. Design 32-33, 477(1996).

[19] Rose, D. V. et al., Physics of Plasmas 6, 4094(1999).

[20] Ottinger, P. F. et al., Phy. of Plasmas 7, 346 (2000).

[21] Tauschwitz, A. et al., Fusion Eng. Design 32-33,493(1996).

[22] Peterson, R. R. et al., eds.,Laser Interaction and Related Plasma Phenomena. AIP Conf. Proc, 406, 251(1997).

[23] Yu, S. et al., Nucl. Instrum. Meth. Phys. Res A 415, 174(1998).

[24] Callahan-Miller,et al.,Nuclear Fusion 39, 883(1999).

[25] Welch, D. R. et al., Physics of Plasmas 9, 2344(2002).

[26] MacLaren, S. A. et al.,Phys. of Plasmas 9, 1712(2002).

[27] Olson, C. L. et al. eds., Heavy Ion Inertial Fusion. AIP Conf. Proc. 152, p. 215. (1986).

[28] Olson, C. L. et al., Fusion Engineering and Design 32-33, 485-491(1996).

[29] S. Eylon et al., Proc. Particle Accel. Conf., (2003).

[30] D. B. Shuman et al., Proc. Particle Accel. Conf., (2003)

[31] E. Henestroza et al., Proc. Particle Accel. Conf. (2003).

[32] E. Henestroza et al., Submitted to Physical Review Special topics - Accelerator and Beams, (2003).

[33] D. Baca et al., Proc. Particle Accel. Conf. (2003).

[34] W. M. Fawley et al. Phys. Plasmas 4,880 (1997).

[35] P.H. Stoltz, et al., Physical Review Special topics Accelerator and Beams, volume 6, 054701 (2003).

[36] R. E. Kirby et al., SLAC-PUB-8212, 2000.

[37] G.F.Dionne, J. Appl. Phys. 44, 5361 (1973). 